\title{
La ingeniería ambiental de la UNESUM, formación universitaria articulada al desarrollo sostenible en el sur de Manabí
}

Jesús Eduardo Alcívar Rivas

\section{Resumen}

Poner en valor a la Universidad actual, es si esta está anclada pertinentemente a un determinado territorio, sin que eso conlleve a desligarse del pensamiento universal; con estos dos elementos en mención, el aporte de este ente académico constituye, por un lado los cambios que provoca en el territorio asentado, y por el otro a la episteme universal. Si ese es el sentido de actuación de la Universidad, provocar y fundamentar cambios en las realidades territoriales, como en el pensamiento universal, de por si se justifica la presencia como en el presente caso en el sur de la provincia de Manabí. Por lo que la Universidad Estatal del Sur de Manabí desde su oferta académica mediante un modelo educativo en la formación profesional, el cómo involucrarse en el territorio que la circunda mediante la vinculación con la sociedad, tomando en cuenta la configuración biofísica-geográfica, y la dinámica económica y social. Además de enmarcarse en la globalización del conocimiento, sin dejar de ajustarse al marco legal vigente. En el contexto en mención, el diseño curricular de la carrera de Ingeniería Ambiental en la Universidad Estatal del Sur de Manabí es donde se inscribe su aporte sustancial desde la actoría de la comunidad universitaria. Profesionales en formación, docentes, autoridades en conexión con la realidad territorial.

Palabras Clave: Universidad, carrera de Ingeniería Ambiental, contexto territorial.

\section{Abstract}

To put in value to the present University, is if this one is anchored pertinently to a determined territory, without that entails to disconnect of the universal thought; with these two elements mentioned, the contribution of this academic entity constitutes, on the one hand, the changes it provokes in the settled territory, and on the other to the universal episteme. If that is the sense of action of the University, provoke and 
ground changes in territorial realities, as in universal thinking, in case the presence is justified as in the present case in the south of the province of Manabí. So that the State University of Southern Manabí from its offer through an educational model in vocational training, how to get involved in the territory that surrounds it by linking with society, taking into account the biophysical-geographic configuration, and the dynamics economic and societal. In addition to framing itself in the globalization of knowledge, if it fails to conform to the current legal framework. In the context mentioned above, the curricular design of the Environmental Engineering course at the State University of Southern Manabí is where its substantial contribution is recorded from the community college actor. Professionals in training, teachers, authorities in connection with the territorial reality.

Keywords: University, Environmental Engineering career, territorial context.

Jesús Eduardo Alcívar Rivas, Ecuador. (eduardoalcivar1956@gmail.com). Msc. en Gestión Ambiental. Director de la Carrera de Ingeniería Ambiental, Universidad del Sur de Manabí, Ecuador - UNESUM.

Fecha de Recepción: 25 de septiembre de 2016. Fecha de aprobación: 25 de noviembre de 2017. 


\section{INTRODUCCIÓN}

El momento actual representa una época de cambios y transformaciones en muchas actividades y procesos. La dinámica dominante alcanza a las Universidades en todo el planeta. Son tiempos de nuevas expectativas, tecnologías y demandas de competencias en los graduados que sobrepasan a las transformaciones ocurridas a veces en cientos de años en otras épocas del saber humano.

Históricamente la Universidad ha sido el lugar de incubación del conocimiento, donde se han formado las bases epistémicas acogida por la sociedad, constituyéndose en ejercicios discursivos adoptados por provenir de los iluminados. En todo este proceso histórico de ensayos y experiencias demostradas y acumuladas, las cuales han sido transmitidas de los pocos iluminados, que además gozaban del prestigio de dominar un bien exclusivo, el conocimiento. La exclusividad del conocimiento en unos pocos llegó a su fin, en la medida que se doto de centros universitarios a todo el planeta, influido por la colonización eurocéntrica de la mano de la iglesia católica, y en la actualidad por la globalización cultural y tecnológica; realidad que requiere la vinculación de la universidad con el mercado de trabajo y humano.

La misión de la Universidad es clara producto de la dominación economicista del mundo, en este contexto se configura la globalización; por lo que el conocimiento deja de lado el universo humano, para anclarse en el universo instrumental, que se concreta en el mercado laboral, el cual no acoge a todos por igual, sino que esta mediado por la competitividad; a esta competitividad dominante del mercado la Universidad no puede, ni debe sucumbir, resistiendo desde el paradigma basado en el bioantropocentrismo.

La dominante instrumentalización hace de la Universidad un espacio de transmisión, dejando de lado la transformación, imbuido en un determinismo de colonización del conocimiento; paradigma que más bien debe alinearse con el dialogo de saberes desde los sentidos de las epistemologías del sur que refiere Boaventura de Sousa, poniendo en valor lo local; que haga del actor profesional en formación un ente productor de conocimiento, sin dejar de dotársele de competencias instrumentales.

La tríada de los procesos sustantivos en la Universidad: formación, investigación y vinculación con la sociedad, no es una entelequia, sino la expresión de una compleja relación con el entorno que debe solucionarse en el mejoramiento social y del territorio. Es el desarrollo como paradigma el fin que puede marcar los objetivos universitarios, pero con una fuerte función social, que debe significar mayor ocupación 
de mano de obra, más aún si esta es calificada; es aquí donde la Universidad debe cumplir la función fundamental del vínculo social con el mercado, donde la oferta académica profesional se ponga a tono con la dinámica económica local, preparando cuadros profesionales de calidad, que no solo sean instrumentos para la producción y productividad, sino generadores de innovación, que a la vez incidan en el cambio de las estructuras socioeconómicas y de gobernanza, fundamentada en la economía social del conocimiento, debido a que esta es libre, publica, común y abierta en acceso igualitario para toda la población, estableciendo una economía colaborativa orientada al bien común, ética y sostenible, creadora de una riqueza común cooperativa basada en la reciprocidad, el mutualismo, la producción entre pares. (FLOK SOCIETY, 2013), para hacer compatible el crecimiento económico con bienestar, donde se apunte a sostener el modelo de desarrollo ya no solo basado en los recursos finitos (que pueden agotarse, incluso los no renovables), sino en los recursos infinitos, los cuales se sustentan en el conocimiento como recurso con carácter estratégico, el que redunde en la producción de nuevos bienes y servicios que rentabilicen los beneficios territoriales.

La transnaciolización de la enseñanza, solucionará el déficit que ocasionará el que muchas Universidades no se adapten a las nuevas circunstancias. Los centros de educación superior que logren responder a las necesidades, ocuparán posiciones de prestigio y recibirán estudiantes transnacionales, con la posibilidad de establecer paradigmas de excelencia. Esos nuevos graduados responderán a las novedades de la tecnología, de los modos de actuación profesionales que se introduzcan en el mercado laboral y por supuesto, estarán en posiciones de avanzadas respecto a los graduados tradicionales.

Consolidación de nuevos esquemas de competencia y cooperación universitaria: la dinámica que se dibuja en el escenario universitario global es la de una mayor presión de la competencia, guiada fundamentalmente por un mercado principal basado en la reputación y el prestigio universitario, asentada en una mayor diferenciación de las universidades y soportada por instrumentos como los "ranking" convertidos en elementos de institucionalización de esa competición.

Para poder competir, las universidades necesitarán también cooperar, porque son pocas las instituciones que puedan afrontar por sí solas y aisladamente los nuevos desafíos y exigencias de esa competición. 
En consecuencia, se espera que las redes de colaboración se refuercen y jueguen un papel aún más decisivo en los próximos años, integrando programas docentes y proyectos de investigación en una misma y específica área.

Irrupción del componente educativo "on-line": la tecnología está conduciendo cambios de gran alcance en el mundo educativo, los recursos docentes están en la Red; las personas demandan estudiar "a la carta", dónde, cuándo y cómo quiere; las experiencias de aprendizaje están dentro y fuera de las aulas.

El desarrollo de este tipo de enseñanzas, por un lado, ampliará el acceso e impulsará el discurso de la internacionalización de una educación superior sin restricciones en el espacio y el tiempo.

Cambios en los esquemas de financiación y organización: la tecnología está facilitando modelos de bajo coste, que serán instrumentados no solamente por el sector privado sino adoptados igualmente por el sector público y los gobiernos; e implica igualmente la adopción de nuevos modelos organizativos y de negocio.

Los liderazgos y decisiones gerenciales se inclinan a ser cada vez más descentralizados, se estimula la educación transnacional, se generan nuevos empleos, funciones y categorías de profesores, se rompe el modelo académico que desarrolla la trayectoria formativa completa en una misma institución y el talento académico se mueve hacia modelos de enseñanza más emprendedores.

La educación como bien público puede verse seriamente amenazada por estas vías y, ante la perspectiva de que los fondos públicos puedan reducirse ocupando su puesto los privados y los pagos de los usuarios, hace necesario reivindicar la decisiva importancia de la financiación de los gobiernos y el carácter de inversión de la educación y la necesidad de encontrar fuentes complementarias y alternativas de financiación.

Las universidades han de responder y tratar de adaptarse a esos escenarios y tendencias que se apuntan en un marco de la educación superior que es ya verdaderamente global. Pero han de afrontar, además, una serie adicional de retos vinculados a su específica situación y circunstancias.

En primer lugar, se debe adaptar la enseñanza a las necesidades sociales. Aún existe una brecha entre la oferta de titulaciones universitarias por un lado y las necesidades sociales y las demandas del sistema productivo por el otro. 
Entre los factores que contribuyen a esa brecha están: un marco institucional que no facilita la adaptación rápida a las demandas sociales; las dificultades para captar la demanda; los procesos de elaboración y desarrollo de los planes; la planificación de la oferta de enseñanzas, que resulta lenta, compleja y burocratizada; el academicismo de muchas instituciones; la escasa flexibilidad y los débiles vínculos con el sistema productivo.

En segundo lugar, es necesario actualizar los sistemas de acreditación y garantía de calidad como uno de los compromisos centrales y de los objetivos universitarios fundamentales.

El problema fundamental actualmente consiste al parecer en que lo formal se impone a lo fundamental, lo interno a lo externo. Los esquemas de garantía de la calidad deben orientarse hacia los aspectos más estratégicos, con menos burocracia evaluativa, con más elementos de diferenciación, con consecuencias más visibles y con mayor conexión hacia el mercado social.

En tercer lugar se deben promover estímulos a la docencia y al profesorado. En especial el profesorado enfrenta desafíos como son la renovación de los métodos docentes, el aumento de tareas, las nuevas dinámicas de interacción con los alumnos y la necesidad de manejar nuevos soportes y lenguajes tecnológicos. Todo ello implica niveles de exigencia cada vez más elevados. Resulta, por ello, imprescindible promover apoyos y estímulos al profesorado para el desarrollo de unas tareas cada vez más diversas y complejas, que requieren superiores dosis de esfuerzo.

En cuarto lugar, es necesario responder a las nuevas expectativas de los estudiantes y atender no solo a una gama de alumnos más plural y heterogénea sino a una tipología de estudiantes nuevos en sus aptitudes, perfiles, habilidades y lenguajes. Para ello resulta necesario entender plenamente a los estudiantes, lograr evitar el desarrollo de programas formativos en los que se incorpora más conocimiento y menos educación. Educación basada en la capacidad de obtener nuevos aprendizajes, de adaptarse a nuevos escenarios, de obtener las competencias y valores que permitan su inserción adecuada en la sociedad.

Para responder a las expectativas de los estudiantes también es necesario atraer y atender al talento en la Universidad. Los casos llamativos de Bill Gates y Steve Jobs, que fueron exitosos hombres de negocios después de abandonar la Universidad, son un ejemplo que expone este problema. La Universidad debe tener la suficiente 
plasticidad para identificar los talentos que llegan a ella y poner la enseñanza en función de sus capacidades.

En quinto lugar se debe valorizar la investigación; la excelencia científica y la competencia emprendedora e innovadora son señas de identidad de las mejores universidades. Para ello se necesita la financiación suficiente y estable, la mejora de las condiciones de trabajo y de la carrera de los investigadores; el impulso de la cooperación entre los agentes del sistema y de la vinculación con el sistema productivo; la superación del déficit de resultados con aplicación productiva; el aumento de la movilidad entre el ámbito académico y el de la empresa; el fortalecimiento de los procesos de transferencia y de generación de empresas y de apoyo al capital riesgo; el impulso de la cultura y el ambiente de innovación y emprendimiento en las universidades; la internacionalización de centros e investigadores para atraer o recuperar a científicos consolidados y la promoción de las vocaciones investigadoras de los jóvenes científicos.

Como una sexta cuestión se debe responder al reto de la eficiencia y eso obligará a hacer más con menos, a mejorar la "función de utilidad social" de las universidades y medir, alcanzar y rendir resultados en docencia, investigación, transferencia, inserción laboral de los titulados, para promover la adecuación entre resultados académicos y demandas sociales.

Junto a la eficiencia y la suficiencia financiera, la equidad constituye un objetivo irrenunciable que cobra su significación más importante en la garantía de que el acceso a la educación no dependa de los niveles de renta y haya posibilidades de acceso a todos los que posean las capacidades para ello.

En séptimo lugar, es necesario mejorar el posicionamiento y la relación con el entorno. El entorno universitario global, y la relación y posición que se ocupa en él, está hoy dominado por unos ranking imposibles de ignorar, difíciles de contener, que se han convertido en árbitros de la excelencia académica universal y que han generado una segmentación del espacio mundial de la educación superior, con universidades de prestigio internacional.

Mejorar las posiciones en ese ranking constituye un objetivo inexcusable para las universidades. Además, la Universidad debe expresar un carácter multidimensional, encargarse de evaluar tanto internamente como lograr la evaluación dentro de los referidos rankings, otras tareas y misiones universitarias relacionadas con la 
docencia, la innovación, la creatividad, la transferencia o el impacto en el entorno académico y social.

Ese impacto en el entorno más próximo ha de constituir igualmente un objetivo destacado de las universidades que, en muchos casos, constituyen un instrumento de indudable relevancia en su entorno institucional, económico y social más cercano y un motor de difusión del conocimiento, desarrollo y progreso a nivel local y regional y que han de protagonizar una fuerte implicación social en su comunidad.

No debe renunciar la universidad, por último, a una dimensión social que es inherente a la educación y a la esencia universitaria de ser fiel, estar comprometida y responder a las sensibilidades de su tiempo y su entorno, constituir un instrumento de desarrollo y bienestar, de creatividad, transmisión de valores, transformación social e igualdad de oportunidades.

La sociedad necesitalugares, comola universidad, de pensamiento, de investigación, de cultura, de ideas renovadas, de ciencia y tecnología que rompa las fronteras del conocimiento, capaces de inocular la aventura de la curiosidad intelectual para imaginar cosas distintas y abrir nuevas dimensiones al conocimiento, el pensamiento y la acción. La universidad ha de responder a esa identidad tradicional e irrenunciable.

\section{La Universidad del Sur de Manabí y su contexto territorial y social}

El modelo educativo de la UNESUM, la sociedad contemporánea lanza retos y desafíos que, en muchos casos, emanan del avance incesante de la ciencia y la tecnología; Esto compromete a las universidades en la búsqueda de respuestas acertadas, a partir de su misión en la formación de profesionales competentes.

El modelo educativo de la UNESUM (2016) se asume como una síntesis de la orientación epistemológica general que sirve de guía a la institución, a sus concepciones filosóficas y sociológicas, así como a los principales enfoques psicopedagógicos en los cuales se basa su plataforma de acción, para dar respuesta a la demanda social. Se reflejan en él las realidades de la universidad, su razón de ser, sus compromisos, su actualidad y perspectivas.

Se ha privilegiado un modelo educativo por competencias con enfoque constructivista, que se organiza sobre la base del perfil de egreso de las carreras. A su vez, este perfil se fortalece mediante los logros y los resultados del aprendizaje, 
alcanzados durante la carrera universitaria, e integrado en los proyectos educativos de saberes.

Este proceso de construcción está centrado en el estudiante y en el aprendizaje, con relación a los recursos didácticos (tecnológicos, analógicos, digitales, etc.) mediados por el docente-facilitador-orientador, y verificado por la evaluación sistemática. Todo esto se ha realizado en un contexto de colaboración constructiva del conocimiento, dentro de un abordaje de planificación con base en los logros del aprendizaje, sin olvidar la investigación, la inter y transdisciplinariedad, y el compromiso social.

De acuerdo con el marco legal vigente, la planificación académica de la UNESUM, partiendo de la autonomía responsable, de calidad y pertinencia, define como dominios académicos la soberanía y seguridad alimentaria; nutrición, salud y bienestar humano; ambiente y biodiversidad; turismo y cultura; soberanía y seguridad alimentaria en el sentido del acceso permanente a los alimentos sanos e inocuos y el acceso a los recursos naturales que permitan una producción sostenible de alimentos por parte de los productores: agua y tierra fundamentalmente. Incluye la comercialización y distribución a través de sistemas justos y solidarios.

Nutrición, salud y bienestar humano como mitigación de los impactos del hambre sobre las sociedades y solución a la realización individual y de las colectividades. El disfrute de sus derechos a la vida y la dignidad.

Las personas dependen de la biodiversidad en su vida cotidiana, la salud humana depende de los bienes y servicios de los ecosistemas (agua dulce, alimentos, fuentes de combustible). La perdida de la biodiversidad empobrece los servicios que prestan los ecosistemas y como consecuencia a la salud humana.

El clima es una parte integral del funcionamiento de los ecosistemas, así mismo la biodiversidad marina. La biodiversidad terrestre está influida por la variabilidad del clima como los fenómenos meteorológicos extremos (sequia e inundaciones) que influyen directamente en salud y productividad del ecosistema.

El turismo cultural es una herramienta de transformación social, consolida los nexos entre los pueblos para su desarrollo, garantizan la permanencia de la identidad y las tradiciones. Proporciona así, un beneficio económico para las comunidades y favorecen la sostenibilidad del patrimonio. El turismo cultural es una prioridad dentro del desarrollo sostenible. 
El contexto territorial y social en que se asienta la UNESUM es la zona de planificación 4 - es en el Pacífico litoral oeste del Ecuador, conformada por las provincias de Manabí y Santo Domingo de los Tsáchilas, configurando 24 cantones, 52 parroquias urbanas y 63 parroquias rurales. El territorio zonal comprende desde el perfil costero hasta las estribaciones de la cordillera occidental de los Andes, en la zona se desarrollan actividades agropecuarias, comerciales y sociales, complementadas mediante una infraestructura sistémica que potencia y fortalece las dinámicas de los territorios y asentamientos humanos.

Los asentamientos humanos más relevantes es el corredor urbano, de: Portoviejo, Santo Domingo y Manta, que juegan un papel protagónico en el desarrollo de la Zona 4. Estas ciudades, sin embargo, poseen índices altos de déficit en servicios básicos residenciales: Portoviejo, con el 80\%; Manta, 61,20\%; y, Santo Domingo de los Tsáchilas, $67 \%$. Además las actividades más importantes, se destacan las de índole turística, agrícola, pesquera y acuícola, así como la ganadería en forma extensiva, sobre todo en el área central norte. (SENPLADES, 2015).

La zona tiene una superficie de $22.717,82 \mathrm{Km}^{2}$, que corresponde al $8.73 \%$ de la superficie total del Ecuador y una población total de 1.780 .717 habitantes al año 2010 , que representa el $12,3 \%$ de la población nacional; de ella, el $50,21 \%$ son hombres y el $49,79 \%$, mujeres. El $60,21 \%$ habita en las áreas urbanas y el $39,79 \%$, en áreas rurales. La tasa de crecimiento anual es de 1,77\% entre 2001 y 2010 , por lo que se encuentra por debajo de la media nacional, de 1,95\%. La densidad poblacional es de $78,38 \mathrm{hab} / \mathrm{Km}^{2}$, cifra que está por encima de la del país, que es de 56,48 hab/Km² (INEC, 2010).

La Población Económicamente Activa (PEA) de la zona es de 663.321 habitantes, es decir, el $37,25 \%$ de la población zonal; el $26,34 \%$ se dedica a la agricultura, la ganadería, la silvicultura y la pesca; el $17,03 \%$, al comercio al por mayor y menor; finalmente, el 7,57\% a la industria manufacturera, (SENPLADES, 2015).

El modelo territorial de la zona 4 se relaciona mediante ejes viales de gran importancia para la producción y el turismo, como la Ruta del Spondylus (perfil del litoral pacífico), Portoviejo-Santo Domingo, Manta-Guayaquil, Manta-PortoviejoQuevedo, Santo Domingo-Quevedo, Santo Domingo-Esmeraldas, Santo Domingo-Quito, Suma-Pedernales y La Concordia-Puerto Nuevo, (SENPLADES, 2015). Los problemas potenciales de la zona se relacionan con la calidad de servicios básicos, calidad de servicios turísticos, valor agregado a la producción 
agropecuaria, manejo sustentable de las cuencas hidrográficas, déficit hídrico, efluentes al mar y ríos que provienen de la industria y los asentamientos humanos, operatividad de la infraestructura, amenazas y vulnerabilidades de origen natural y antrópico e indicadores sociales con déficit de cobertura y calidad en territorios con alta población rural, vocación agropecuaria, de acuicultura y pesca, ubicación estratégica y roles de sus asentamientos humanos para desarrollar infraestructura logística y de transporte, (SENPLADES, 2015).

Para superar estos problemas se requiere de políticas públicas adecuadas y de acertados procesos de planificación, de planes, programas y proyectos para la gestión ambiental, la investigación y la vinculación con la colectividad, para ejecutar acciones de asesoramiento, manejo y utilización adecuada de los recursos naturales, siendo prioridad para el Estado contar con instituciones de educación superior como la UNESUM, que preparen profesionales de calidad en diversas áreas del conocimiento y particularmente en el área de la ciencias ambientales, que contribuyan a orientar el accionar antrópico y su relación con la naturaleza.

El área de influencia directa de las provincias de Manabí y Santo Domingo de los Tsáchilas, presenta características particulares en relación al resto del país, y se caracterizan por estar vinculadas a la producción agrícola, ganadera e industrial, actividades que no escapa de la problemática de contaminación ambiental.

En la provincia de Manabí existen sectores que permanentemente son afectados por factores antrópicos y naturales, como largos periodos de sequía, que son consecuencias de la ampliación de la frontera agrícola, que implica una agresiva desforestación de las especies nativas para la extracción de madera y la implementación de cultivos, afectando la biodiversidad y los recursos naturales y que incide en el deterioro de la calidad del suelo, agua, aire y a la población en general.

La Provincia de Manabí cuenta con una diversidad biológica que se caracteriza por la presencia de variados ecosistemas con multiplicidad de especies vegetales y animales, el patrimonio se está desapareciendo aceleradamente. En efecto, el deterioro de hábitats, la sobre explotación de las especies terrestres y marinas, la contaminación de estuarios y humedales, requieren de una atención permanente.

Manabí cuenta con una singular diversidad de ambientes naturales, se destacan el Parque Nacional Machalilla, y los bosques protectores Pacoche, y Chongón 
Colonche (sur provincial) y la Reserva Ecológica Mache Chindul. También existen ecosistemas de extrema sensibilidad ambiental como humedales, manglares y estuarios que, podrían significar importantes ingresos económicos para la provincia. Sumados a estos atributos naturales y paisajísticos, se debe sumar la riqueza arqueológica, la identidad manabita y el conocimiento ancestral de prácticas agrícolas y culturales como oportunidades que permitirían afirmar la identidad provincial.

La particularidad topográfica de la provincia, hace que drene sus aguas hacia la costa del Pacífico. En su recorrido sufren una acelerada contaminación que requiere del aporte técnico y responsable para minimizar el deterioro del recurso hídrico de la provincia. Por su apreciable volumen de almacenaje y producción de agua, las cuencas hidrográficas tienen potencial de generación de energía hidroeléctrica en las presas Poza Honda, la Esperanza, Daule Peripa y represa Carrizal Chone en fase de terminación. La vocación agrícola de sus suelos y la variedad climática de la región, favorece el buen desarrollo agrícola, pecuario e industrial.

El servicio de alcantarillado en la provincia es alarmante, solo está presente en 16 cabeceras cantonales en tan solo el $45 \%$ en el área urbana con una cobertura del $50 \%$, y de 1,7\% en el área rural. Ello conduce a que las descargas se realicen en quebradas, ríos, playas, mar, constituyéndose en puntos focales de contaminación y genera impactos ambientales al agua, al suelo, al aire y a los seres humanos, (SENPLADES, 2015).

La caracterización descrita es contratendencial al incremento sostenido y exponencial de las áreas silvestres protegidas en todo el mundo, en donde existen unos 10.000 territorios resguardados a nivel global, entre parques nacionales, santuarios ecológicos, humedales protegidos, territorios indígenas o tribales, áreas marítimas sumando un total aproximado de 18 millones de $\mathrm{Km}^{2}$ En el Ecuador el Sistema Nacional de Áreas Protegidas (SNAP) representa el 19\% del territorio nacional, que en superficie se aproxima a $50 \mathrm{mil} \mathrm{km}^{2}$ el que alberga la potencialidad biodiversa, como la hídrica; lo que contrasta con el déficit de la provincia de Manabí, la que tan solo cuenta con un $6 \%$ de su superficie en la categoría de áreas protegidas. (MAE, 2010); desafío no solo de la gobernanza nacional y provincial, sino de la Universidad en su incremento y manejo sostenido. 
La vinculación con sociedad de la UNESUM, constituye, en el contexto ecuatoriano, una de las funciones sustantivas del quehacer universitario, expresada claramente en la Ley Orgánica de Educación Superior.

La Universidad Estatal del Sur de Manabí (UNESUM), actualmente se encuentra en un proceso de cambio institucional, fortaleciendo la formación de los talentos humanos, a nivel de docentes, investigadores y estudiantes de las distintas carreras. También en la investigación científica y tecnológica orientada a construir alternativas tecnológicas para resolver los problemas que afectan a su entorno y el país.

La UNESUM promueve la vinculación con la sociedad, en coherencia con los principios de pertinencia académica y pertinencia social. El diagnóstico de la vinculación universitaria con la sociedad identificó los siguientes elementos problemáticos:

- Débil articulación de la UNESUM con el entorno.

- Falta de articulación de los proyectos de vinculación con los planes de desarrollo territorial a nivel de provincia, cantón y parroquias.

- Talento humano con limitada cultura sobre articulación de la academia con la sociedad.

- Débil armonización del conocimiento científico, saber ancestral y las vivencias y experiencias de las comunidades.

- Poca disponibilidad de infraestructura y recursos materiales (equipos e instrumentos) para la realización de la capacitación y difusión del conocimiento.

- Deficiente articulación entre la investigación y la formación profesional en grado y posgrado.

- Incipiente desarrollo de los Centros de Capacitación y Desarrollo Local (CECADEL) como efectivos centros de capacitación continua y transferencia de tecnología que dinamicen la vinculación de la academia con la sociedad.

Marco legal para la vinculación con la sociedad, la vinculación con la sociedad desde la UNESUM, tiene como basamento legal diferentes documentos legales y normativos. En primer lugar, tiene en cuenta el nivel constitucional a partir del artículo 35 de la Constitución del Ecuador, donde dice: "El sistema de educación 
superior tiene como finalidad la formación académica y profesional con visión científica y humanista; la investigación científica y tecnológica; la innovación, promoción, desarrollo y difusión de los saberes y las culturas; la construcción de soluciones para los problemas del país, en relación con los objetivos del régimen de desarrollo". Partir de ahí, se puede señalar como otros elementos del marco legal, la ley orgánica de la educación superior (LOES) en sus artículos:

Articulo. 24. Distribución de los recursos.

Artículo. 107.- Principio de pertinencia.

Artículo 2d.- Regular la gestión académica-formativa en todos los niveles de formación y modalidades de aprendizaje de la educación superior...

Artículo 77.- Pertinencia de las carreras y programas académicos.

Artículo 78.- Fortalezas o dominios académicos de las instituciones de educación superior.

Artículo 82.- Vinculación con la sociedad y educación continúa.

Las actividades de vinculación con la sociedad se enmarcan en lo establecido en el artículo 9 del Reglamento de Carrera y Escalafón del Personal Académico, que prescribe textualmente lo siguiente:

Artículo 9.- Actividades de vinculación con la sociedad.

La carrera de ingeniería ambiental, también cuenta como base legal, los objetivos del plan de vinculación de la UNESUM.

\section{Objetivo general:}

Contribuir al desarrollo sostenible de la zona sur de Manabí, la región y el país, en base a la articulación de la UNESUM con empresas privadas, instituciones públicas, organizaciones sociales, gobiernos autónomos descentralizados e instituciones de educación superior; así como, la coordinación de las prácticas pre-profesionales y pasantías, seguimiento a graduados, educación continua y el fortalecimiento de los centros de capacitación y desarrollo local, promoviendo convenios y acuerdos con los distintos actores, en la perspectiva de generar impactos económicos, sociales, ambientales y organizacionales. 
Objetivos especificos:

Articular a la UNESUM con otras instituciones de educación superior, empresas privadas, instituciones públicas, organizaciones sociales y gobiernos autónomos descentralizados en la perspectiva de impulsar el desarrollo sostenible del entorno local, regional y nacional.

Coordinar las prácticas pre-profesionales y pasantías para fortalecer la formación del talento humano como agentes de cambio.

El seguimiento a los graduados para establecer la pertinencia entre la contribución de la UNESUM y los requerimientos de los actores del entorno.

Promover la educación continua del talento humano para la actualización permanente en los avances de la ciencia y tecnología; así como, la difusión del conocimiento.

Ampliar los servicios de capacitación, asistencia técnica, consultoría y proyectos de desarrollo a través de los centros de capacitación y desarrollo local, orientados a generar impactos productivos, organizacionales y sociales, respetando la cultura e idiosincrasia de los pueblos y conservando los recursos naturales.

La carrera de Ingeniería ambiental en la UNESUM, esta se adscribe a todos los principios del marco legal, objetivos y políticas que asume la UNESUM en lo referido a la vinculación con el territorio en su apuesta por el desarrollo sostenible. Como documento normativo y estrategia de trabajo, se cuenta con el diseño curricular de la carrera, donde se definen los objetivos principales de formación y los ejes principales de los procesos sustantivos a ejecutar dentro de la carrera (Alcívar, 2016).

En este diseño curricular, ante todo se establece la vinculación de la formación del profesional con el Plan Nacional del Buen Vivir, extendido a lo que corresponde territorialmente en las definiciones de la Agenda Zonal 4, en el marco normativo vigente desde la categoría constitucional. Entre las estrategias que se incorporan en primer lugar está la estrategia 7.2.c. del PNBV. Constituye una guía para que la carrera desarrolle mecanismos integrales de prevención, monitoreo, control y/o erradicación de especies invasoras, para precautelar la salud pública y la protección de los ecosistemas y su biodiversidad, particularmente de las especies nativas, endémicas y en peligro de extinción. Se requiere por eso de profesionales 
ambientales con conocimientos científicos para implementar mecanismos, técnicas y tecnologías apropiadas para la garantía del patrimonio natural y su remediación.

De igual manera, la estrategia 7.2.d. es un enunciado para impulsar el análisis de paisajes y la interacción socioeconómica del territorio en los procesos de planificación y de ordenamiento territorial, de manera articulada entre los diferentes niveles de Gobierno. A estos efectos, el profesional en ingeniería ambiental es capaz de asesorar técnicamente el desarrollo de los planes de ordenamiento territorial en las diferentes entidades gubernamentales a fin de garantizar mediante la innovación técnica, tecnológica y científica los aprendizajes necesarios para valorar, conservar e implementar la sustentabilidad.

La estrategia 7.2.e, permite a los profesionales reconocer, respetar y promover los conocimientos y saberes ancestrales, las innovaciones y las prácticas tradicionales sustentables de las comunidades, pueblos y nacionalidades para fortalecer la conservación y el uso sustentable de la biodiversidad, con su participación plena y efectiva.

La estrategia 7.2.k. se orienta a promover el acceso a fondos nacionales e internacionales para el financiamiento de la conservación del patrimonio natural, mediante programas integrales y ambiciosos y la estrategia 7.2.m. sustenta fomentar la investigación y los estudios prospectivos sobre el uso sustentable y la conservación de la biodiversidad terrestre, acuática, marina-costera; lo que implica que la carrera debe formar profesionales ambientales que estén en capacidad de formular, planificar, ejecutar, evaluar y gestionar proyectos multidisciplinarios de cooperación nacional e internacional, con el propósito de fortalecer la investigación, la vinculación y el intercambio científico, académico y tecnológico para la conservación del patrimonio natural.

Estrategia 7.6.g. Establecer un registro de descargas de aguas residuales por sectores, en afluentes, para regular, controlar y sancionar la contaminación del recurso hídrico, así como desarrollar acciones específicas para su tratamiento y reposición de agua de calidad.

La carrera de Ingeniería Ambiental está comprometida a formar técnicos con dominios en uso y tratamiento de agua potable de calidad para consumo humano, que contribuya a mejorar la calidad del buen vivir de los habitantes, así mismo en el tratamiento y uso de aguas residuales. 
Estrategia 7.6. h. Controlar y regular de manera integral el uso y la importación de sustancias químicas peligrosas especialmente mercurio, cianuro, asbesto y contaminantes orgánicos persistentes, como medida para reducir la contaminación ambiental y proteger la salud de las personas. Los profesionales estarán en capacidad de promocionar y aplicar las normativas del manejo de los contaminantes del agua, suelo y aire.

Estrategia 7.6.m. Reforzar e incentivar el tratamiento de aguas residuales de uso doméstico, industrial, minero y agrícola, a fin de disminuir la contaminación en los sitios de descarga y de cumplir con las normas, regulaciones y estándares de calidad ambiental. La generación de tecnologías limpias será una alternativa que contribuya al mejoramiento y reutilización de las aguas residuales.

Estrategia 7.6.k. Fortalecer los mecanismos y las capacidades institucionales nacionales y locales para prevenir y controlar la contaminación de aire, suelo y agua, así como para garantizar la reparación integral de los daños y pasivos socio ambientales que se generen. El profesional producto de la carrera de Ingeniería Ambiental ofrecerá asesoramiento técnico de prevención, control y remediación a la contaminación del aire, suelo y agua.

El Cambio Climático y el Medio Ambiente es un tema de actualidad científica y geopolítica mundial que ha concitado el interés de todos los gobernantes, diplomáticos, militares y religiosos del universo por la gravedad de sus efectos catastróficos que están asolando y destruyendo al Planeta Tierra y a sus habitantes. El cambio climático es la modificación del clima con respecto al historial climático a una escala global o regional. Tales cambios se producen a muy diversas escalas de tiempo y sobre todos los parámetros meteorológicos: temperatura, presión atmosférica, precipitaciones, nubosidad, etc. En teoría, son debidos tanto a causas naturales como antropogénicas. Por "cambio climático" se entiende un cambio de clima atribuido directa o indirectamente a la actividad humana que altera la composición de la atmósfera mundial y que se suma a la variabilidad natural del clima observada durante períodos comparables.

El cambio climático y la agricultura son procesos relacionados entre sí, ya que ambos tienen escala global. Se proyecta que el calentamiento global tendrá impactos significativos que afectaran a la agricultura, la temperatura, dióxido de carbono, deshielos, precipitación y la interacción entre estos elementos. Estas condiciones determinan la capacidad de carga de la biosfera para producir suficiente alimento para todos los humanos y animales domesticados. El efecto global del cambio climático en la agricultura dependerá del balance de esos efectos. El estudio 
de los efectos del cambio climático global podría ayudar a prevenir y adaptar adecuadamente el sector agrícola para maximizar la producción de la agricultura. Los pueblos indígenas serán los primeros en sentirse afectados por el cambio climático, ya que su supervivencia depende de los recursos naturales de su entorno, y cualquier cambio, como por ejemplo sequías extremas, pueden amenazar su vida. Por la disminución del agua estos pueblos pierden su terreno cultural y forma de vida por generaciones, donde múltiples culturas han creado formas sociales, culturales y artísticas en torno al ecosistema, causando un desplazamiento de pueblos indígenas a ciudades desarrolladas.

Se cree que el sector agropecuario es uno de los principales emisores de gases y al igual que los efectos del uso de tierras, pueden producir el cambio climático. Además de ser un importante usuario de tierras y consumidor de combustibles fósiles, la agricultura y ganadería contribuyen directamente a las emisiones de gases de efecto invernadero por medio de técnicas en el cultivo como la producción de arroz y la cría de ganado, según el Panel Intergubernamental del Cambio Climático, los combustibles fósiles, el uso de tierras, y la agricultura, son las tres causas principales del incremento de los gases de efecto invernadero desde hace 250 años.

Las causas principales de cambio climático son las causas naturales que incluyen actividad volcánica o cambios en la energía recibida desde el sol, entre otro; y las causas antrópicas generadas por actividades humanas, que incluye la quema de combustibles fósiles, tala y quema de bosques, entre otros, y que desde la Revolución Industrial ha influido la emisión de $\mathrm{CO} 2$ y otros gases que han ayudado a amplificar el efecto invernadero natural que se refleja en el calentamiento global. El Ecuador es un país vulnerable por su fragilidad por el cambio climático, dependiendo de la región, porque tiene relación directa con la exposición a la amenaza, a la sensibilidad, al clima y a la capacidad de adaptación que se presenta en cada una de éstas. La Sierra y la Amazonía son más vulnerables a deslizamientos; en cambio en la Costa, se presentan más inundaciones y subidas del nivel del mar, mientras que la sequía es más frecuente en zonas de la Sierra y la Costa.

Ante este fenómeno de vulnerabilidad el Ministerio del Ambiente ha emprendido planes y proyectos relacionados con la gobernabilidad del agua, es decir, proteger las cuencas hidrográficas existentes en el país; disminuir la vulnerabilidad social, económica y ambiental para responder y resistir los impactos a través de acciones, mitigación y concienciación que conduzcan a la lucha contra el cambio climático 
a nivel mundial; poner énfasis en la seguridad alimentaria; y evitar al máximo la deforestación.

Los efectos del cambio climático en Ecuador tienen relación con: Las precipitaciones decrecieron en el noroccidente ecuatoriano, pero cuando ocurren se dan con intensidad y en pocas horas; es más notorio el incremento de períodos fríos en el noroccidente del país y noches más cálidas en la costa sur y la región meridional del Oriente muestra incrementos de temperatura, tanto para el día como para la noche.

Ecuador es un país megadiverso, rico en ecosistemas en todas las regiones del país, y variedad agro productiva, los cuales han estado sujetos a presiones económicas que ha ocasionado grandes problemas relacionados con la degradación de la tierra, la desertificación, y acrecentados por los efectos de la sequía. Se estima que alrededor del $47 \%$ del territorio nacional presenta problemas de degradación de la tierra debido a causas provenientes de las actividades humanas y naturales.

La Convención de las Naciones Unidas de lucha contra la Desertificación (CNULD), y la comunidad internacional han reconocido a la desertificación, la degradación de la tierra y la sequía como graves problemas que trascienden las fronteras de los países y cuyos efectos inciden en todas las regiones del mundo.

La Convención Marco de las Naciones Unidas sobre el Cambio Climático (CMCC) define a este fenómeno como "un cambio de clima atribuido directa o indirectamente a la actividad humana que altera la composición de la atmósfera mundial y que se suma a la variabilidad natural del clima observada durante períodos".

Lo cierto es que el mundo ha empezado a sentir los efectos del cambio climático: eventos extremos, cambios bruscos de temperatura, disminución de glaciares, entre otros, y todos causados por el hombre. Los científicos se han puesto de acuerdo que a partir de la revolución industrial la cantidad de gases contaminantes incrementó y eso ocasiona que aumente la temperatura global que altera el clima y afecta a las especies.

El Gobierno de Ecuador es el primero país en reconocer el cambio climático, y a través del Ministerio del Ambiente presentó la Estrategia Nacional de Cambio Climático 2012-2025, como una herramienta de trabajo que busca integrar distintos sectores y redoblar esfuerzos para conservar los recursos forestales, manglares y páramos, apoyar y fomentar la eficiencia energética, mejorar las prácticas 
ambientales y reducir el consumo de los recursos. En esta estrategia aparece el cambio de la matriz energética, con la construcción de hidroeléctricas que convertirá al país en un consumidor de energía limpia, así mismo la línea de sustitución de las cocinas a gas.

El país tiene todo un marco regulatorio al respecto. La Constitución, por ejemplo, reconoce los derechos de la naturaleza. Ecuador es uno de los pocos países de la región que hace referencia explícita a la temática del cambio climático en su Carta Magna; mientras que, el Plan Nacional del Buen Vivir (2013-2017) tiene como séptimo objetivo "garantizar los derechos de la naturaleza y promover la sostenibilidad ambiental territorial y global".

La política 7.10 está orientada a implementar medidas de mitigación y adaptación al cambio climático para reducir la vulnerabilidad económica y ambiental.

El Ministerio del Ambiente estará encargado de la formulación y ejecución de la estrategia nacional y el plan que permita generar e implementar acciones y medidas tendientes a concienciar en el país la importancia de la lucha contra este proceso natural y antropogénico y que incluyan mecanismos de coordinación y articulación interinstitucional.

"El cambio climático es una amenaza fundamental para el desarrollo", declaró, estimando que los gobiernos "tienen que actuar ya" para limitar el calentamiento del planeta y financiar políticas que propicien un desarrollo sostenible. Este año la comunidad internacional puede y debe encontrar maneras de financiar las intervenciones sobre el cambio climático y el desarrollo Estrategia 7.10.d. del PNBV: Incorporar criterios de mitigación y adaptación al cambio climático en la formulación y evaluación de planes y proyectos estratégicos, así como en los planes de contingencia que puedan afectar la infraestructura y la provisión de servicios.

El Ingeniero Ambiental estará en capacidad de ofertar sus servicios a las industrias locales generadoras de contaminantes atmosféricos como monóxido de carbono, dióxido de carbono, trióxido de carbono y ácido sulfhídrico, óxido de nitrógeno, que son los más perjudiciales para el ambiente.

Estrategia 7.10.k. Fortalecer la participación nacional en las negociaciones internacionales de cambio climático lograr mayor efectividad en la gobernanza ambientaly en el cumplimiento de los compromisos parala tecnología, la consolidación de una nueva arquitectura financiera y la transferencia de recursos financieros por parte de los países industrializados, como compensación a los efectos negativos 
del cambio climático en los países no industrializados. El Ingeniero Ambiental contribuye a desarrollar investigaciones ambientales utilizando las energías limpias que ayudan a mitigar los efectos negativos del cambio climáticos y divulgando sus resultados en eventos locales, nacionales e internacionales. Otra de las actividades que incide en el cambio climático es la explotación petrolera, con frecuentes derrames de petróleo en los bosques, mares, ríos, que provocan contaminación ambiental y que se refleja en daños a la fauna marina, aves, vegetación y aguas; de igual forma los productos de desechos gaseosos expulsados en las refinerías ocasionan la alteración, no sólo de la atmósfera, sino también de las aguas, tierra, vegetación, aves y otros animales.

\section{CONCLUSIONES}

En el mundo actual la enseñanza universitaria se ha convertido en un proceso dinámico que necesita del entorno social y territorial en que se encuentra para su propio desarrollo. Para eso se deben fortalecer los procesos de investigación y vinculación con la sociedad, como principales fuentes de transformación de la formación académica. La Universidad es un actor social que tiene un rol específico en su entorno y sus estrategias y normas deben incluir estas funciones. En el diseño de la carrera de Ingeniería ambiental de la UNESUM, se han tenido en cuenta estas condicionantes y se mantiene el carácter renovador como elemento importante de la investigación, la vinculación con la sociedad y como resultado la formación del profesional.

\section{BIBLIOGRAFÍA}

- Alcívar Rivas J. E. (2016). Diseño de la carrera de Ingeniería Ambiental. Documento de trabajo Universidad Estatal del Sur de Manabí.

- Constitución de la República del Ecuador, 2008.

- Convención de las Naciones Unidas de Lucha contra la Desertificación (CNULD, UNCCD. 1994).

- Boaventura de Sousa Santos (2009). Una Epistemología del Sur. La reinvención del conocimiento y la emancipación social. Buenos Aires: Siglo XXI Editores, CLACSO.

- FLOK Society (2013). BUEN CONOCER. Modelos sostenibles y políticas públicas para una economía social del conocimiento común y abierto en Ecuador. 
- INEC. (20010). Censos de Población y Vivienda.

- Ley Orgánica de Educación Superior, 2010. (Ecuador)

- Ministerio del Ambiente, (2010). Sistema Nacional de Áreas Protegidas.

- SENPLADES. (2013 - 2017). Plan Nacional del Buen Vivir.

- SENPLADES. (2015). Agenda Zonal 4. Provincias Manabí y Santo Domingo de los Tsáchilas. Quito-Ecuador: Secretario Nacional de Planificación y Desarrollo.

- UNESUM. (2016). Modelo Educativo de la UNESUM. Documento de trabajo. Universidad Estatal del Sur de Manabí.

- UNESUM. (2015). Plan de vinculación con la sociedad de la Universidad Estatal del Sur de Manabi (2016-2021). Documento de trabajo Universidad Estatal del Sur de Manabí.

- Vázquez García J. A. (2015). Nuevos escenarios y tendencias universitarias. RIE. 33 (1). 13-26. 